\title{
Novel Human Acute Ischemic Stroke Blood Clot Analogs for In Vitro Thrombectomy Testing
}

\author{
(D) S.T. Fitzgerald, (D)Y. Liu, (DD. Dai, (D) O.M. Mereuta, (D). Abbasi, (D).L.A. Larco, (D)A.S. Douglas, (DD.F. Kallmes, (D). Savastano, \\ (D) K.M. Doyle, and (DW. Brinjikji
}

\begin{abstract}
BACKGROUND AND PURPOSE: Previous studies have successfully created blood clot analogs for in vitro endovascular device testing using animal blood of various species. Blood components vary greatly among species; therefore, creating clot analogs from human blood is likely a more accurate representation of thrombi formed in the human vasculature.
\end{abstract}

MATERIALS AND METHODS: Following approval from the Mayo Clinic institutional review board, human whole-blood and platelet donations were obtained from the blood transfusion service. Twelve clot analogs were created by combining different ratios of red blood cells + buffy coat, plasma, and platelets. Thrombin and calcium chloride were added to stimulate coagulation. Clot composition was assessed using histologic and immunohistochemical staining. To assess the similarities of mechanical properties to patient clots, 3 types of clot analogs (soft, elastic, and stiff) were selected for in vitro thrombectomy testing.

RESULTS: The range of histopathologic compositions produced is representative of clots removed during thrombectomy procedures. The red blood cell composition ranged from $8.9 \%$ to $91.4 \%$, and fibrin composition ranged from $3.1 \%$ to $53.4 \%$. Platelets (CD42b) and von Willebrand Factor ranged from $0.5 \%$ to $47.1 \%$ and $1.0 \%$ to $63.4 \%$, respectively. The soft clots had the highest firstpass effect and successful revascularization rates followed by the elastic and stiff clots. Distal embolization events were observed when clot ingestion could not be achieved, requiring device pullback. The incidence rate of distal embolization was the highest for the stiff clots due to the weak clot/device integration.

CONCLUSIONS: Red blood cell-rich, fibrin-rich, and platelet-rich clot analogs that mimic clots retrieved from patients with acute ischemic stroke were created in vitro. Differing retrieval outcomes were confirmed using in vitro thrombectomy testing in a subset of clots.

ABBREVIATIONS: $\mathrm{A}=$ aspiration; $\mathrm{AIS}=$ acute ischemic stroke; $\mathrm{DA}=$ direct aspiration; FPE $=$ first-pass effect; $\mathrm{MSB}=$ Martius Scarlett Blue; RBC $=$ red blood cell; $S R=$ successful revascularization; STR $=$ Stent retriever; $\mathrm{vWF}=$ von Willebrand factor; $\mathrm{WBC}=$ white blood cell

I the treatment of acute ischemic stroke (AIS), the achievement of complete revascularization from a single mechanical thrombectomy attempt, termed first-pass effect (FPE), is associated with significantly improved outcomes for patients. ${ }^{1,2}$ Removing the clot in a fragmented manner increases the potential of embolization to new territories, a major contributing factor to poor neurologic outcomes due to additional brain infarction. ${ }^{3-5}$ Despite the advancement in the second-generation mechanical thrombectomy devices, the rates

Received September 29, 2020; accepted after revision January 26, 2021.

From the Departments of Radiology (S.T.F., Y.L., D.D., O.M.M., M.A., D.F.K., W.B.)

and Neurosurgery (J.L.A.L., L.S., W.B.), Mayo Clinic, Rochester, Minnesota; and

Department of Physiology (S.T.F., O.M.M., A.S.D., K.M.D.) and SFI Centre for

Research in Medical Devices (O.M.M., A.S.D., K.M.D.), National University of Ireland Galway, Galway, Ireland.

This work was supported by the National Institutes of Health grant No. R01 NS105853, the European Regional Development Fund and Science Foundation Ireland grant No. 13/RC/2073, and Enterprise Ireland (IP20190865).

Paper previously presented, in part, as a poster presentation at: International Stroke Conference, February 18-21, 2020; Los Angeles, California. of FPE remain low, as low as $29 \%$ in the recently reported Contact Aspiration vs Stent Retriever for Successful Revascularization (ASTER) trial. ${ }^{6}$

Previous studies have demonstrated that a wide variety of occlusive clots can cause large-vessel occlusion, ${ }^{7-11}$ and clot composition has been shown to have a significant impact on the success of mechanical thrombectomy procedures. ${ }^{7,12,13}$ These findings suggest that to further advance the success rates of stroke intervention, we must turn our attention to clot composition and compare

Please address correspondence to Seán Fitzgerald, PhD, Department of Physiology, Human Biology Building, National University of Ireland Galway, Galway, H91 TK33, Ireland; e-mail: Sean.Fitzgerald@nuigalway.ie; @FitzSeanT; @WBrinjikji

\footnotetext{
- Indicates open access to non-subscribers at www.ajnr.org

$\equiv$ Indicates article with online supplemental data.

Indicates article with supplemental on-line videos.

http://dx.doi.org/10.3174/ajnr.A7102
} 
Table 1: Volume of components added to each clot analog type $^{a}$

\begin{tabular}{lccc}
\hline Ratio & $\begin{array}{c}\text { Platelets } \\
(\mu \mathrm{l})\end{array}$ & $\begin{array}{c}\text { Plasma } \\
(\mu \mathrm{l})\end{array}$ & $\begin{array}{c}\text { RBC + Buffy Coat } \\
(\mu \mathrm{l})\end{array}$ \\
\hline Plasma only & & & \\
1:5 & 2400 & 600 & \\
1:10 & 0 & 2700 & 300 \\
1:50 & 0 & 2940 & 60 \\
1:100 & 0 & 2970 & 30 \\
Platelets only & & & \\
1:5 & 2400 & 0 & 600 \\
1:10 & 2700 & 0 & 300 \\
1:50 & 2940 & 0 & 60 \\
1:100 & 2970 & 0 & 30 \\
Plasma and & & & \\
platelets & & & 600 \\
1:5 & 1200 & 1200 & 300 \\
1:10 & 1350 & 1350 & 60 \\
1:50 & 1470 & 1470 & 30 \\
1:100 & 1485 & 1485 &
\end{tabular}

${ }^{\mathrm{a}}$ Three microliters of thrombin $(1 \mathrm{NIH} / \mathrm{mL})$ and $300 \mu \mathrm{L}$ of $5 \% \mathrm{CaCl}_{2}$ were added to stimulate coagulation.

treatment strategies using in vitro thrombectomy models of the cerebral vasculature. Previous studies have successfully created blood clot analogs for in vitro testing using animal blood of various species, which have significantly advanced our understanding of clot biomechanics and imaging characteristics. ${ }^{13-19}$ However, blood components and blood groups vary among species; ${ }^{20}$ thus, creating clot analogs with human blood is likely a more accurate representation of thrombi formed in the human vasculature.

The hypothesis of the study was that the diverse range of clots retrieved from patients with AIS can be accurately replicated using human blood by mimicking the process by which clots form in vivo. The rationale for this study is that because the success of mechanical thrombectomy procedures is influenced by the composition of the clot, creating human clot analogs that accurately represent the different phenotypes retrieved from patients and testing them in an in vitro thrombectomy system will allow us to compare the performance of different thrombectomy devices and techniques. We will be able to determine the optimum treatment approach for each clot phenotype, thereby optimizing the chances of achieving the desired first-pass TICI 3 outcome in the clinical setting. ${ }^{1}$ To assess the similarities of mechanical properties to patient clots, we selected 3 types of clot analogs (soft, elastic, and stiff) for in vitro thrombectomy testing.

\section{MATERIALS AND METHODS}

\section{Human Clot Analog Creation}

This study received institutional review board approval from Mayo Clinic Rochester in accordance with the ethical standards of the Declaration of Helsinki. A total of 12 clot analog types were created as per Table 1 . These clots analogs were selected to be representative of the previously identified phenotypes of clots retrieved from patients with AIS, including red blood cell (RBC)rich, fibrin-rich, and platelet-rich clots. ${ }^{21}$

A human whole-blood donation and a human platelet donation from 2 separate donors were obtained from the Mayo Clinic blood transfusion service. The whole blood was centrifuged at $1200 \mathrm{rpm}$ for 20 minutes at $20^{\circ} \mathrm{C}$ to separate it into its constituents. $^{22}$ Plasma was harvested by pipetting, and the remaining RBCs and buffy coat were mixed together by inverting. Plasma and platelets were combined first as per Table 1; then, $3 \mu \mathrm{L}$ of thrombin (1 NIH/mL, No. T6884; Roche Diagnostic) was added to activate platelets for a total of 1-2 minutes while continuously mixing. Three hundred microliters of $5 \%$ calcium chloride $\left(\mathrm{CaCl}_{2}\right.$, No. C1016; Sigma Aldrich) solution was then added followed by the RBCs + buffy coat mixture. The tube was then quickly mixed by inversion 5 times and then, the clot analog mixture was drawn into a $3 \mathrm{~mL}$ syringe. The syringes were spun overnight at $20 \mathrm{rpm}$ at room temperature to mimic the dynamic flow conditions of the human vasculature.

\section{Patient Cohort}

Clots were collected from 100 patients who underwent mechanical thrombectomy for the treatment of AIS at Mayo Clinic Rochester. When greater than 1 procedural pass was needed to retrieve the occlusive clot, all fragments of clot were combined for histologic analysis. The inclusion criteria were patients 18 years of age or older and having undergone mechanical thrombectomy treatment for AIS with clot material available for analysis. A waiver of informed consent was granted for the purposes of collecting retrieved clot material from patients with AIS for this study.

\section{Histologic Processing and Staining}

Gross photos were taken of each clot and analog before fixation overnight in $10 \%$ phosphate-buffered formalin. All clots and analogs were then processed using a standard tissue-processing protocol and embedded in paraffin. The formalin-fixed paraffin-embedded material was cut into $3-\mu \mathrm{m}$ sections. The Martius Scarlett Blue (MSB) stain is now regarded as the criterion standard for assessing clot composition because it identifies platelet-rich regions of thrombi in addition to RBCs, white blood cells (WBCs), and fibrin. ${ }^{21,23}$ Two representative slides were stained with MSB to identify the common clot constituents: RBCs, WBCs, fibrin, platelets/ other, collagen, and calcification as described previously. ${ }^{21,23}$ Clot phenotypes for both the clinical samples and the clot analogs were defined on the basis of the dominant component (in percentages) in each clot as determined by the MSB histologic staining.

\section{Immunohistochemistry}

Platelet and von Willebrand factor (vWF) levels are useful additional hallmarks of clot composition. ${ }^{24-26}$ Immunohistochemical staining for platelets (CD42b) and the vWF was performed on a Bond RX autostainer (Leica Biosystems). Antigen retrieval with Tris-EDTA was performed for platelet staining (anti-CD42b); no antigen retrieval was used for vWF staining. For the primary antibody (antiCD42b, ab27669, 1:200 dilution [Abcam]; anti-vWF, A-0082, 1:200 dilution [Dako]), the incubation time was 30 minutes. Negative controls were performed by omission of the primary antibody step. A Bond Polymer Refine Red Detection system (Leica Biosystems), which incorporates a post-primary antibody, polymer reagent, Fast Red chromogen and hematoxylin counterstain, was used for visualization. Sections were washed in warm soapy water, dehydrated in increasing alcohol gradients, cleared in xylene, and mounted with DPX Mountant (Sigma-Aldrich). 
RBC+Buffy Coat: Plasma Only

1:5

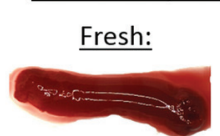

|cmin| Init|mul|uí

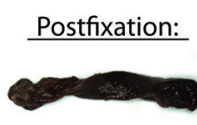

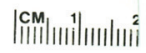

1:10

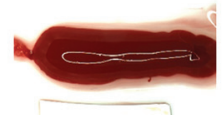

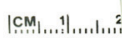

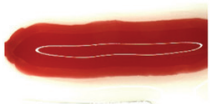

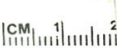

1:100

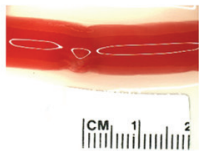

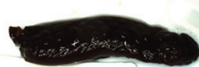

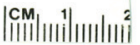

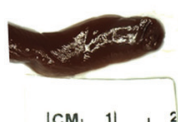

|cMilmi||m|m|mii

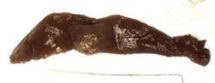

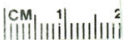

RBC + Buffy Coat: Platelets Only
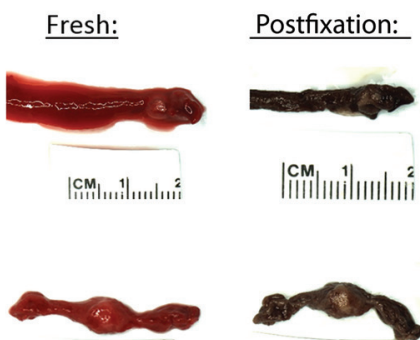

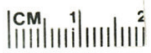

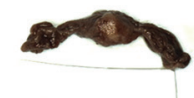

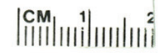
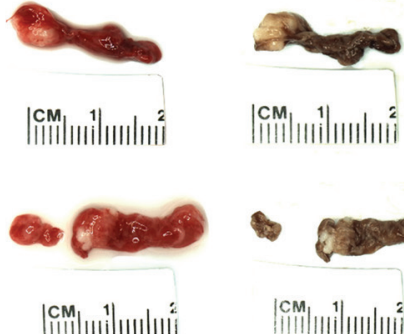

RBC+Buffy Coat: Plasma+Platelets
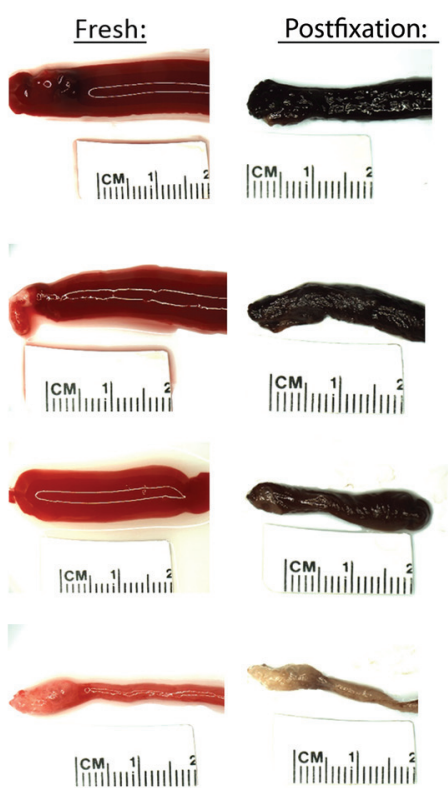

FIG 1. Gross photographs of human blood clot analogs pre- and postfixation. Gross photographs of human blood clot analogs of various compositions were obtained pre- and postfixation in 10\% neutral buffered formalin. Clot analogs were created using different ratios (1:5, 1:10, 1:50, and 1:100) of RBC+ buffy coat and plasma only, platelets only, and plasma + platelets.

\section{Imaging and Quantification}

Following staining, a representative slide of each stain was scanned at 20x magnification (EasyScan Pro; Motic Digital Pathology). Histologic quantification was performed on the digital slide using Image Analysis Software (Orbit; www.Orbit.bio) as described previously. ${ }^{21,25}$ The percentage area of each component (RBC, WBC, fibrin, and platelet/other) within the clot was calculated for the histologic staining with $\mathrm{MSB}^{21}$ The percentage area of positive immunohistochemistry staining was calculated separately for CD42b and vWF. ${ }^{25}$

\section{Thrombectomy Testing in a Benchtop Stroke Platform}

The mechanical properties of clots vary on the basis of their histologic composition; clot analogs with an increasing volume of platelets contract to a greater degree due to the force of platelet contraction, resulting in stiffer clot analogs. ${ }^{27}$ Clot analogs that have a high RBC content will typically be softer, more friable clots, and clots made from plasma only will produce clot analogs with a network of thin fibrin strands. Three phenotypes of clot analogs with varying compositions of RBCs, plasma, and platelets were selected to represent prominent phenotypes of clots retrieved from patients with AIS during thrombectomy: soft (1:10 RBCs+buffy coat : plasma only), elastic (1:5 RBCs+buffy coat : platelets + plasma), and stiff (1:10 RBCs + buffy coat : platelets + plasma).

Thrombectomy testing was performed on these clots inside a benchtop stroke platform as previously described. ${ }^{28,29}$ Briefly, a cerebrovascular glass model, in which the lumen resembles the intracranial internal carotid artery, the anterior cerebral artery, and the middle cerebral arteries, is connected to a customized flow system to deliver flow with a physiologically representative flow rate and pressure. Clot analogs measuring $6 \mathrm{~mm}$ in length were introduced into the flow system and embolized to the M1-M2 bifurcation.
Revascularization was performed using the following: 1) the direct aspiration (DA) technique with the Sofia $6 \mathrm{~F}$ aspiration catheter (MicroVention), and 2) stent retriever with aspiration (stent retriever $+\mathrm{A}$ ) technique with the Solitaire stent retriever (Medtronic) and the Sofia 6F aspiration catheter. For each type of clot analog and revascularization technique, 5 clot analogs were made to replicate large-vessel occlusion stroke. For each largevessel occlusion case, 3 device passes were attempted before declaring failure. Revascularization results, number of passes, and embolization events were recorded for each test.

Ingestion was defined as complete ingestion of the clot into the catheter, FPE was defined as complete removal of the clot from the target artery in the first procedural pass, successful recanalization (SR) was defined as the complete removal of the clot from the target artery within 3 procedural passes, and distal embolization was defined as the occurrence of visible fragments of clots being dislodged and migrating distally from the target vessel. The thrombectomy processes were recorded, and the failure mechanisms, including the presence of distal embolization, were confirmed following the procedure.

\section{Statistical Analysis}

All statistical correlations were assessed, and graphs were generated using GraphPad Prism 8 (GraphPad Software). MSB histologic composition was reported as the percentage of the total clot area. Positive immunohistochemistry staining (CD42b and vWF) was reported as the percentage of the total clot area. A ShapiroWilk test indicated that quantitative variables did not follow a standard normal distribution. The nonparametric Spearman $\rho$ correlation was used to assess the similarity between clot analogs and clinical samples. A level of statistical significance for all analyses was set at $P<0.05$. 


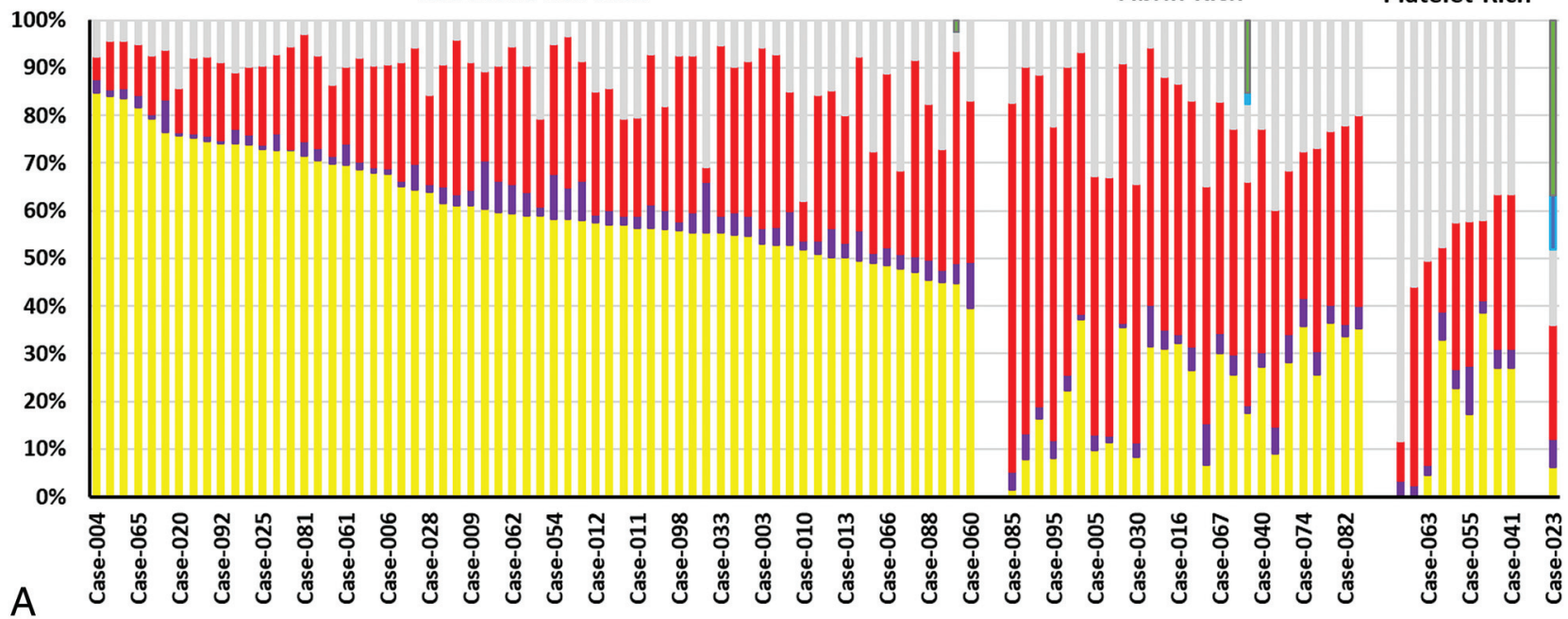

\section{Clot Analog MSB Quantification}

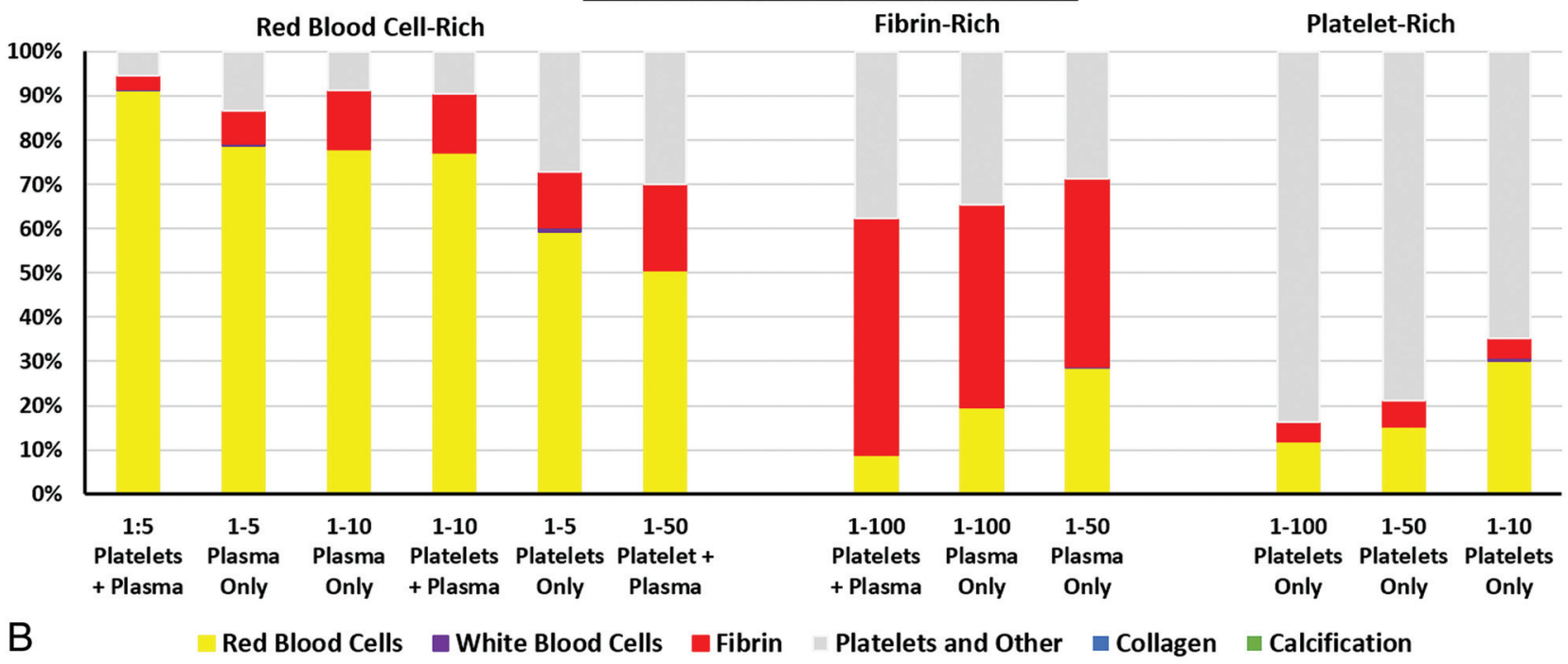

FIG 2. Histologic quantification of MSB-stained human clots retrieved from patients and human clot analogs created in vitro. A, The histologic composition of 100 various clots retrieved from patients was assessed using the MSB stain and grouped according to their dominant component, resulting in 3 main groups; RBC-rich, fibrin-rich, and platelet-rich. B. The histologic composition of various human clot analogs created in vitro was also assessed using the MSB stain and grouped according to their dominant component, resulting in the same 3 phenotypes; RBC-rich, fibrin-rich, and platelet-rich.

\section{RESULTS}

\section{Clot Analog Appearance}

The gross appearance of each of the human clot analogs after clot formation and also postfixation in 10\% neutral buffered formalin is shown in Fig 1. Clot analogs that are rich in RBC clots (eg, 1:10 RBCs + buffy coat : plasma only) are dark red after creation and black postfixation. Clots that contain high platelet content (eg, 1:100 RBCs+buffy coat : platelets only) have white platelet-rich regions that are visible both pre- and postfixation. Clots that are fibrin-rich but not platelet-rich are light red after creation and brownish postfixation (eg, 1:50 RBCs + buffy coat : plasma only). The platelet-rich clots are smaller in clot volume due to the effect of platelet-stimulated contraction of the clots. The clot analogs produced have a gross appearance similar to that of clots extracted from patients during mechanical thrombectomy procedures for the treatment of AIS.

\section{Histologic Composition}

The MSB stain was used to assess the histologic composition of the clot analogs (Online Supplemental Data) and of the clots retrieved from patients with AIS (Fig 2). RBC-rich, fibrin-rich, and platelet-rich clot analogs that mimic clots retrieved from patients with AIS were created. The range of histopathologic compositions of the clot analogs is similar to that of the clinical samples (Fig 2). The addition of a large volume of RBCs leads to an RBC-rich clot regardless of whether platelets and/or plasma were added (Fig 2). The RBC composition of the clot analogs ranged from $8.9 \%$ to $91.4 \%$, and the clots retrieved from the patients ranged from $<1 \%$ to $85 \%$. There was a significant positive correlation between the RBC composition of the analogs and the clinical samples $\left(r_{\mathrm{s}}=\right.$ $0.755, P=.010^{*}$ ). The platelet composition of the clot analogs ranged from $5.4 \%$ to $83.7 \%$, while the clinical samples ranged from $3 \%$ to $88 \%\left(r_{\mathrm{s}}=0.618, P=.048^{\star}\right)$. Fibrin composition of the clot 
analogs ranged from $3 \%$ to $53 \%$ and from $3 \%$ to $77 \%$ in the clinical samples $\left(r_{\mathrm{s}}=0.136, P=.694\right)$. WBCs are typically a minor component of clots and account for an average of 3.5\% of clinical clots and $1 \%$ of clot analogs $\left(r_{\mathrm{s}}=0.311, P=.345\right)$. Each of these components is in line with the composition of AIS clots reported previously in the literature. ${ }^{7,21,30}$

\section{Immunohistochemical Composition}

The composition of platelets (CD42b) varied from $0.5 \%$ to $47.1 \%$ of the total area, and the composition of vWF varied from $1.1 \%$ to $63.4 \%$ of the total area (Table 1). Clot analogs made with platelets only had the largest proportion of both CD42b and vWF present, while clot analogs made with plasma only had the lowest levels of both platelets and vWF. Clot analogs containing both platelets and plasma had moderate levels of both platelets and vWF present. An example of a clot analog closely resembling a clot retrieved from a patient with AIS is shown in Fig 3.

\section{Revascularization Results}

The 3 types of clot analogs are associated with different revascularization outcomes (Table 2). The soft clots are associated with the highest ingestion, FPE, and successful recanalization rates,

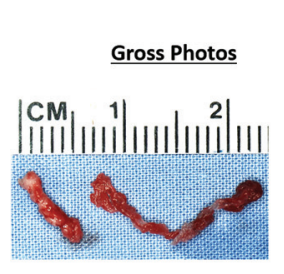

A

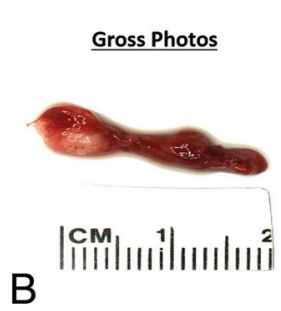

Clot Retrieved from Patient with AIS:

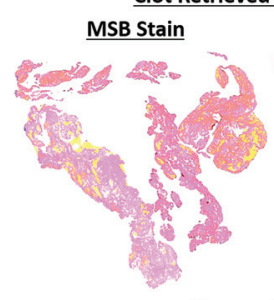

Human Clot Analog:

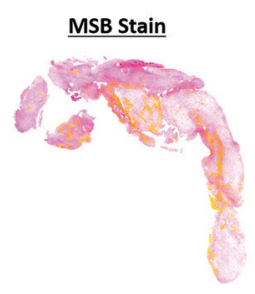
Platelets (CD42b)

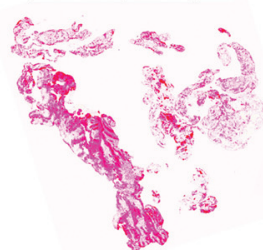

Platelets (CD42b)

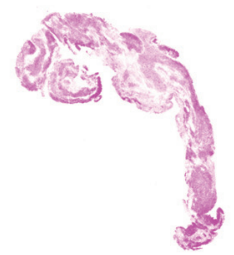

followed by the elastic and stiff clots. The rate of distal embolization increased as the rate of ingestion decreased.

All of soft clots could be successfully removed with 1 pass, and no distal embolization was observed. By means of the DA technique, $80 \%$ (4 of 5) of clots were ingested (Supplemental Online Video S1), with 1 exception in which the catheter tip was corked by the clot. Using the stent retriever + A technique, all the clots could be pulled out without any distal embolization.

For the elastic clot and using the DA technique, only $40 \%$ (2 of 5 ) of the clots could be ingested and the other 3 clots were corked by the suction catheter and pulled out. During catheter pull, the clot was elongated under the tensional load applied by the vacuum suction and the pressure gradient across the clot. As the clot was moved to the internal carotid artery terminus, a temporary nearto-total obstruction of the flow increased the antegrade pressure gradient and eventually fractured the clot and caused distal embolization (Supplemental Online Video S2). In a similar fashion, the stent retriever + A technique also fractured the clot during device pull, causing distal embolization (Supplemental Online Video S3). Distal embolization of clot fragments is the main reason for repeat passes to recanalization.

For the stiff clots and using the DA technique, only 20\% (1 of 5 ) of the clots could be ingested. During device pull, $80 \%$ (4 of 5) of the clots lost integration with the suction catheter due to the antegrade pressure gradient and resulted in failed revascularization. The integration of the clots to the stent retriever was stronger than the aspiration catheter alone, resulting in a lower distal embolization rate and higher revascularization rate (Table 2).

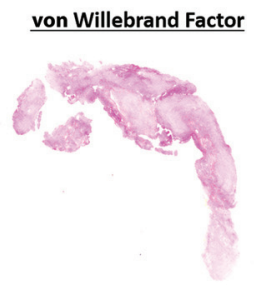

FIG 3. Histology and immunohistochemical-stained images of clots retrieved from a patient with AIS and of a human clot analog. A comparison of the similarity in terms of gross photographs, MSB stain, CD42b (platelets), and VWF between a clot retrieved from a patient with AIS $(A)$ and a human clot analog (1:50 RBC + buffy coat/platelets) is shown (B). MSB stain: RBCs (yellow), WBCs (purple), fibrin (red), platelets/other (grey), and collagen (blue). Platelets (CD42b) and vWF; positive staining is red.

Table 2: In vitro thrombectomy results

\begin{tabular}{lccccc}
\hline Clot Type & Ingestion Rate & FPE Rate & SR Rate & Passes to Achieve SR & DE Rate \\
\hline Soft & & & & & \\
DA & $80 \%$ & $100 \%$ & $100 \%$ & 1.0 & 0 \\
STR + A & NA & $100 \%$ & $100 \%$ & 1.0 & 0 \\
Elastic & & & & & $20 \%$ \\
DA & $40 \%$ & $100 \%$ & $100 \%$ & 1.0 & $60 \%$ \\
STR + A & NA & $40 \%$ & $100 \%$ & 1.8 & $80 \%$ \\
Stiff & & & & & $40 \%$ \\
DA & $20 \%$ & $40 \%$ & $40 \%$ & 1.0 & 1.3 \\
STR + A & NA & $60 \%$ & $80 \%$ &
\end{tabular}

Note:-DE indicates distal embolization; STR + A, stent retriever + aspiration; NA, not applicable.

\section{DISCUSSION}

In this study, a range of novel in vitro human clot analogs that mimic the gross appearance and histologic composition of clots retrieved from patients with AIS was created. The composition of the clot analogs was confirmed using the MSB histologic stain for the main components and immunohistochemical staining for the identification of platelets and vWF. Furthermore, a subset of clot analogs were tested in an in vitro thrombectomy model and demonstrated that revascularization outcome is related to both the composition of the clots and the technique used to retrieve them. The results of this study are important because they prove that human clot analogs that accurately replicate the histologic composition of clots retrieved from patients can be created, and they demonstrate that these clot analogs can be used in an in vitro thrombectomy setup to compare the performance of 
different treatment approaches, potentially leading to a clinical benefit for the patients.

The inability of second-generation thrombectomy aspiration and stent retriever devices to dramatically improve the rates of FPE following endovascular treatment of AIS suggests that the effect is not specifically device-related. ${ }^{6}$ An understanding of clot histologic characteristics and recanalization outcomes is potentially of great importance in improving device selection and device development. There is a growing awareness of the importance of clot phenotypes, mechanical properties, clot-device interactions, and the interactions of clots with the surrounding vessel, factors that influence revascularization rates. ${ }^{1,27,31-34}$ However, clinicians largely continue to treat patients using their preferred treatment strategy rather than tailoring their treatment strategy to suit the suspected clot composition because there have been few clinical studies comparing various thrombectomy techniques in their ability to retrieve different phenotypes of thrombi from patients with AIS. ${ }^{35}$ The histologic composition of the clot analog phenotypes created in this study is in line with the range of clot compositions typically seen in AIS. ${ }^{21}$ Clots retrieved from patients can generally be stratified into 3 main phenotypes based on their histologic composition: RBC-rich, fibrin-rich, and platelet-rich. Each of these phenotypes was successfully replicated in vitro in this study.

The novel clot analogs described herein used in conjunction with human vascular replicator systems that can accurately replicate the intracranial vasculature, cardiac cycle, and intracranial blood pressure may enable the optimization of techniques and treatment strategies. A recent study using similar human clot analogs in an in vitro thrombectomy system also demonstrated that the composition of the clot analogs significantly affects the outcome of the procedure. ${ }^{36}$ By assessing the success rate of all of the various thrombectomy devices and techniques in retrieving different phenotypes of clots as described, we can arrive at a better understanding of how to improve the rates of FPE. The use of human clot analogs and accurate in vitro thrombectomy systems could be a valuable training resource for educating physicians on the potential clinical significance of tailoring their treatment strategy to optimize their chances of achieving complete revascularization. In addition to their use in in vitro thrombectomy testing, human clot analogs can also be used to investigate the ability of novel diagnostic imaging methods to identify the composition of the occlusive clot. ${ }^{37,38}$

For the DA technique, the FPE is associated with successful clot ingestion and depends on the clot mechanical properties. Of the 15 clots tested, FPEs were achieved for 12 clots, and 7 (58\%) of them were due to successful ingestion. The ingestion rates were $80 \%, 40 \%$, and $20 \%$ for the soft, elastic, and stiff clots, respectively. Clots with a higher composition of platelets and fibrin have a higher stiffness and friction coefficient, ${ }^{17,39}$ making them difficult to deform into the catheter tip and be ingested. For the 8 clots without successful ingestion, 6 (75\%) of them presented with distal embolization and resulted in repeat device passes or failed revascularization. Suction catheters that can generate a large suction force to deform the clots and overcome the clot friction to ingest the clots could be beneficial. ${ }^{40}$ However, the thrombectomy tests are performed in a glass phantom, and the arterial response to suction was not captured. Under suction, the vessel could collapse due to the reduced intraluminal pressure and evacuation of fluid, hypothetically related to the more severe vessel injuries using suction catheters rather than the stent retrievers. ${ }^{41}$ The safety profile of the new-generation large-bore suction catheters needs to be further validated.

When we compared the stent retriever + A technique with the DA technique, the FPE rate was the same (100\%) for the soft clots, lower (40\% versus 100\%) for the elastic clots, and higher (60\% versus $40 \%$ ) than the DA technique for the stiff clots, showing a clear relation with the clot mechanical properties. For both the DA and the stent retriever $+\mathrm{A}$ techniques in which the FPE was not achieved, the failure mechanism was the poor clot/device integration with downstream migration of emboli, similar to that found in a whole human brain thrombectomy platform. ${ }^{42}$ During clot retrieval by device pull (stent retriever or suction catheter without clot ingestion), the clot/device integration has to fight against the tensional force generated by the device and the antegrade pressure gradient. Compared with the DA technique in which the clot was engaged with the suction catheter only at the clot "head" (Supplemental Online Video S2), in the stent retriever + A technique, the clot integration was stronger as the clot was grabbed by the stent tines along the clot length (Supplemental Online Video S3). This result could be the reason for higher FPE and successful revascularization rates for the stiff clot analogs. On the other hand, the clot/stent integration was still weak, with multiple passes needed to revascularize. Future stent technologies should enable better clot integration, especially for stiff clots.

This study has some limitations. First, the whole-blood and platelet donations were not collected from the same patient, and the blood phenotypes of each were not available. Second, blood phenotype has been shown to impact coagulation, and this method may need to be adjusted slightly for each blood phenotype. Finally, the thrombectomy tests were performed in a glass phantom, and the arterial response to suction was not captured.

\section{CONCLUSIONS}

RBC-rich, fibrin-rich, and platelet-rich clot analogs that mimic clots retrieved from patients with AIS were created in vitro. Differing retrieval outcomes were confirmed using in vitro thrombectomy testing in a subset of clots. The use of human clot analogs and accurate in vitro thrombectomy systems could be a valuable training resource for physicians to optimize their chances of achieving complete revascularization for every clot phenotype.

Disclosures: Sean T. Fitzgerald-RELATED: Grant: National Institutes of Health, Comments: This work was supported by the National Institutes of Health grant No. R01 NS105853; the European Regional Development Fund and Science Foundation Ireland grant No. 13/RC/2073; and Enterprise Ireland (IP20190865).* Yang Liu-RELATED: Grant: National Institutes of Health, Comments: R01 NS105853 to Waleed Brinjikji*; UNRELATED: Employment: Mayo Clinic, Rochester; Stock/Stock Options: Endovascular Engineering Inc, Comments: Endovascular Engineering Inc is developing thrombectomy devices, and I serve on the Scientific Advisory Board, paid by stock options. Oana Madalina Mereuta -RELATED: Grant: National Institutes of Health, the European Regional Development Fund and Science Foundation Ireland, Comments: This work was supported by the National Institutes of Health grant No. R01NS105853, the European Regional Development Fund, and Science Foundation Ireland (grant No. 13/RC/2073).* Andrew S. Douglas-RELATED: Grant: National Institutes of Health, the European Regional Development Fund and Science Foundation 
Ireland, and Enterprise Ireland, Comments: This work was supported by the National Institutes of Health grant No. R01 NS105853, the European Regional Development Fund and Science Foundation Ireland grant No. 13/RC/2073, and Enterprise Ireland (IP20190865).* David F. Kallmes-RELATED: Grant: National Institutes of Health: National Institute of Neurological Disorders and Stroke, Comments: R01 NS105853*; UNRELATED: Board Membership: Triticum, Vesalio, Comments: Advisory Board, Data and Safety Monitoring Board*; Grants/Grants Pending: Cerenovus, MiVi Neurovascular*; Patents (Planned, Pending or Issued): Mayo Clinic*; Stock/Stock Options: Marblehead Medical, LLC, Superior Medical Experts, LLC. Karen M. Doyle-RELATED: Grant: Science Foundation Ireland, Comments: This work was supported by the European Regional Development Fund and Science Foundation Ireland grant No. 13/RC/2073*; UNRELATED: Employment: National University of Ireland Galway; Grants/Grants Pending: Cerenovus.* Waleed Brinjikji-RELATED: Grant: National Institutes of Health: National Institute of Neurological Disorders and Stroke.* *Money paid to the institution.

\section{REFERENCES}

1. Zaidat OO, Castonguay AC, Linfante I, et al. First pass effect. Stroke 2018;49:660-66 CrossRef Medline

2. Nikoubashman O, Dekeyzer S, Riabikin A, et al. True first-pass effect. Stroke 2019;50:2140-46 CrossRef Medline

3. Todo A, Minaeian A, Sahni R, et al. Incidence and outcome of procedural distal emboli using the Penumbra thrombectomy for acute stroke. J Neurointerv Surg 2013;5:135-38 CrossRef Medline

4. Kurre W, Vorlaender K, Aguilar-Pérez M, et al. Frequency and relevance of anterior cerebral artery embolism caused by mechanical thrombectomy of middle cerebral artery occlusion. AJNR Am J Neuroradiol 2013;34:1606-11 CrossRef Medline

5. Jindal G, Carvalho HD, Wessell A, et al. Beyond the first pass: revascularization remains critical in stroke thrombectomy. J Neurointerv Surg 2019;11:1095-99 CrossRef Medline

6. Ducroux C, Piotin M, Gory B, et al; ASTER Trial Investigators. First pass effect with contact aspiration and stent retrievers in the Aspiration versus Stent Retriever (ASTER) trial. J Neurointerv Surg 2020;12:386-91 CrossRef Medline

7. Hashimoto T, Hayakawa M, Funatsu N, et al. Histopathologic analysis of retrieved thrombi associated with successful reperfusion after acute stroke thrombectomy. Stroke 2016;47:3035-37 CrossRef Medline

8. Liebeskind DS, Sanossian N, Yong WH, et al. CT and MRI early vessel signs reflect clot composition in acute stroke. Stroke 2011;42:1237-43 CrossRef Medline

9. Maegerlein C, Friedrich B, Berndt M, et al. Impact of histological thrombus composition on preinterventional thrombus migration in patients with acute occlusions of the middle cerebral artery. Interv Neuroradiol 2018;24:70-75 CrossRef Medline

10. Saver JL, Goyal M, Bonafe A, et al; SWIFT PRIME Investigators. Stent-retriever thrombectomy after intravenous t-PA vs. t-PA alone in stroke. $N$ Engl J Med 2015;372:2285-95 CrossRef Medline

11. Staessens S, Denorme F, François O, et al. Structural analysis of ischemic stroke thrombi: histological indications for therapy resistance. Haematologica 2020;105:498-507 CrossRef Medline

12. Boeckh-Behrens T, Schubert M, Förschler A, et al. The impact of histological clot composition in embolic stroke. Clin Neuroradiol 2016;26:189-97 CrossRef Medline

13. Chueh JY, Wakhloo AK, Hendricks GH, et al. Mechanical characterization of thromboemboli in acute ischemic stroke and laboratory embolus analogs. AJNR Am J Neuroradiol 2011;32:1237-44 CrossRef

14. Preut A, Laughlin M, Jensen $H$, et al. Novel method for emboli ana$\log$ formation towards improved stroke retrieval devices. J Biomech 2018;80:121- 28 CrossRef Medline

15. Duffy S, Farrell M, McArdle K, et al. Novel methodology to replicate clot analogs with diverse composition in acute ischemic stroke. $J$ Neurointerv Surg 2017;9:486-91 CrossRef Medline

16. Ren M, Lin Z-J, Qian H, et al. Embolic middle cerebral artery occlusion model using thrombin and fibrinogen composed clots in rat. $J$ Neurosci Methods 2012;211:296-304 CrossRef Medline
17. Gunning GM, McArdle K, Mirza M, et al. Clot friction variation with fibrin content; implications for resistance to thrombectomy. $J$ Neurointerv Surg 2018;10:34-38 CrossRef Medline

18. Bretzner M, Lopes R, McCarthy R, et al. Texture parameters of R2* maps are correlated with iron concentration and red blood cells count in clot analogs: a 7-T micro-MRI study. J Neuroradiol 2020;47:306-11 CrossRef Medline

19. Gonzalez AV, Buerke B, Görlich D, et al. Clot analog attenuation in non-contrast CT predicts histology: an experimental study using machine learning. Transl Stroke Res 2020;11:940-49 CrossRef Medline

20. Stormont CJ. Blood groups in animals. J Am Vet Med Assoc 1982;181:1120-24 Medline

21. Fitzgerald ST, Wang S, Dai D, et al. Platelet-rich clots as identified by Martius Scarlet Blue staining are isodense on NCCT. J Neurointerv Surg 2019;11:1145-49 CrossRef Medline

22. Van Wie BJ, Sofer SS. Experimental considerations for the centrifugal separation of blood cell components. Int J Artif Organs. 1986;9(1):4958 Medline

23. Staessens S, Fitzgerald S, Andersson T, et al. Histological stroke clot analysis after thrombectomy: technical aspects and recommendations. Int J Stroke 2020;15:467-76 CrossRef Medline

24. Staessens S, Denorme F, François O, et al. Dense fibrin, von Willebrand factor and extracellular DNA are specific structural hallmarks of platelet-rich areas in ischemic stroke thrombi. In: Proceedings of the Congress of the International Society on Thrombosis and Haemostasis. Melbourne, Australia. July 6-10, 2019

25. Douglas A, Fitzgerald S, Mereuta OM, et al. Platelet-rich emboli are associated with von Willebrand factor levels and have poorer revascularization outcomes. J Neurointerv Surg 2020;12:557-62 CrossRef Medline

26. Mereuta OM, Fitzgerald S, Abbasi M, et al. Abstract WP268: von Willebrand factor expression in various subtypes of acute ischemic stroke. Stroke 2020;51:AWP268 https://www.ahajournals.org/ doi/10.1161/str.51.suppl_1.WP268. Accessed March 06, 2020

27. Johnson S, Chueh J, Gounis MJ, et al. Mechanical behavior of in vitro blood clots and the implications for acute ischemic stroke treatment. J Neurointerv Surg 2020;12:853-57 CrossRef Medline

28. Reddy AS, Liu Y, Cockrum J, et al. Construction of a comprehensive endovascular test bed for research and device development in mechanical thrombectomy in stroke. J Neurosurg 2020;1:1-8 CrossRef Medline

29. Liu Y, Zheng Y, Reddy AS, et al. Analysis of human emboli and thrombectomy forces in large-vessel occlusion stroke. J Neurosurg 2020;1:1-9 CrossRef Medline

30. Ye G, Gao Q, Qi P, et al. The role of diabetes mellitus on the thrombus composition in patients with acute ischemic stroke. Interv Neuroradiol 2020;26:329-36 CrossRef Medline

31. Eng W, Kim M, Pham S, et al. Micromechanics of blood clots. Arteriosclerosis, Thrombosis, and Vascular Biology 2018;38(Suppl 1) CrossRef

32. van der Marel K, Chueh JY, Brooks OW, et al. Quantitative assessment of device-clot interaction for stent retriever thrombectomy. $J$ Neurointerv Surg 2016;8:1278-82 CrossRef Medline

33. Fitzgerald S, Mereuta OM, Doyle KM, et al. Correlation of imaging and histopathology of thrombi in acute ischemic stroke with etiology and outcome. J Neurosurg Sci 2019;63:292-300 CrossRef Medline

34. Sporns PB, Jeibmann A, Minnerup J, et al. Histological clot composition is associated with preinterventional clot migration in acute stroke patients. Stroke 2019;50:2065-71 CrossRef Medline

35. Mohammaden MH, Haussen DC, Camara Cp D, et al. Hyperdense vessel sign as a potential guide for the choice of stent retriever versus contact aspiration as first-line thrombectomy strategy. J Neurointerv Surg 2020 Jul 31. [Epub ahead of print] CrossRef Medline

36. Fitzgerald S, Ryan D, Thornton J, et al. Preclinical evaluation of Millipede 088 intracranial aspiration catheter in cadaver and in vitro thrombectomy models. J Neurointerv Surg 2020 Jun 30. [Epub ahead of print] CrossRef Medline

37. Liu HC, Abbasi M, Ding YH, et al. Characterizing thrombus with multiple red blood cell compositions by optical coherence tomography 
attenuation coefficient. J Biophotonics 2020 Dec 31. [Epub ahead of print] CrossRef Medline

38. Liu HC, Abbasi M, Ding YH, et al. Characterizing blood clots using acoustic radiation force optical coherence elastography and ultrasound shear wave elastography. Phys Med Biol 2021;66:035013 CrossRef Medline

39. Liu Y, Reddy AS, Cockrum J, et al. Standardized fabrication method of human-derived emboli with histologic and mechanical quantification for stroke research. J Stroke Cerebrovasc Dis 2020;29:105205 CrossRef Medline
40. Fitzgerald S, Ryan D, Thornton J, et al. Preclinical evaluation of Millipede 088 intracranial aspiration catheter in cadaver and in vitro thrombectomy models. J Neurointerv Surg 2020 Jun 30. [Epub ahead of print] CrossRef Medline

41. Gory B, Bresson D, Kessler I, et al. Histopathologic evaluation of arterial wall response to 5 neurovascular mechanical thrombectomy devices in a swine model. AJNR Am J Neuroradiol 2013;34:2192-98 CrossRef Medline

42. Gebrezgiabhier D, Liu Y, Reddy AS, et al. A human brain test bed for research in large vessel occlusion stroke. J Neurosurg 2021 Jan;22:1-9 CrossRef Medline 